\title{
La resolución de problemas a través de trabajos prácticos de laboratorio como estrategia para el aprendizaje de conceptos químicos en estudiantes de décimo grado de Educación Media
}

\author{
Problem Solving through Practical Laboratory Work, \\ as Strategy for Chemical Learning Concepts in \\ Students of Tenth degree Middle School
}

Paola Alexandra Moya Peralta ${ }^{1}$

\begin{abstract}
Resumen
El presente trabajo corresponde a una propuesta investigativa en la cual se desarrollaron trabajos prácticos de laboratorio a través de la resolución de problemas. Se abordaron los contenidos correspondientes a los empleados por el Colegio Mayor de San Bartolomé y de manera contextualizada a su entorno. Este proyecto se realizó con estudiantes de grado décimo de dicha institución y se buscó despertar el interés hacia el aprendizaje de la química, ya que los trabajos de laboratorio se convirtieron en una estrategia para desarrollar en ellos una serie de habilidades científicas. La idea fue comenzar de una forma básica hasta una forma más compleja, que permitió relacionar, de manera adecuada, la teoría, la práctica y la resolución de problemas cotidianos.
\end{abstract}

Este proyecto se inició en el segundo semestre de 2014 con una caracterización de las estrategias de enseñanza que los estudiantes tuvieron en el área de ciencias y las estrategias de interés por parte de ellos para el periodo 2015. En el año en curso se aplicaron las situaciones problema guiadas por una pregunta problema que debían solucionar los estudiantes desde la teoría y la práctica, y finalmente se determinaron los niveles de competencias científicas que fortalecieron los estudiantes con la propuesta.

Licenciada en Química Universidad Pedagógica Nacional. Correo electrónico: dqu_pmoya711@pedagogica. edu.co paoale711@hotmail.com 


\section{Palabras claves}

Resolución de problemas, actitudes, trabajo de laboratorio, enseñanza, ciencia.

\section{Abstract}

This work corresponds to a proposal in which practical laboratory work was developed across the resolution of problems, it approached the contents corresponding to the handled ones for the institution and contextualized way to their environment. It developed with students of tenth degree of Colegio Mayor de San Bartolomé, sought to wake the interest up towards the learning of the chemistry, where the laboratory works turned a strategy to develop in them a series of scientific skills. It was begun of a basic form up to a more complex form, which allowed to relate in a suitable way, the theory, the practice and the resolution of daily problems.

This project began in the second semester of 2014 with a characterization of the strategies of education that the students had in the area of sciences and the strategies of interest on the part of them for the period 2015, already in the year in process the situations applied problem to themselves guided for a question problem that the students had to solve from, the theory and the practice. Levels of scientific expertise acquired students were determined with the proposal.

\section{Keywords}

Resolution of problems, attitudes, labs practices, education, science.

\section{Introducción}

La enseñanza de la química es importante porque a través de esta los estudiantes aprenden conceptos que les posibilitan la interpretación de los fenómenos que ocurren a su alrededor. Sin embargo, muchas investigaciones educativas orientadas a la enseñanza de las ciencias han permitido demostrar diversos problemas en diferentes ámbitos, como la visión de la ciencia desde un aprendizaje memorístico, que conducen a la rutina.
La actitud del estudiante hacia el aprendizaje de las ciencias incide de manera directa sobre el proceso de enseñanza- aprendizaje (Galindo, 2011). Por esta razón, las prácticas de laboratorio se convierten en una actividad esencial para la enseñanza de la química, porque promueve habilidades científicas desde las más sencillas hasta las más complejas. Así mismo, la resolución de problemas es una manera que motiva al estudiante a solucionar, desde su conocimiento teórico-práctico, situaciones problema reales.

Este proyecto tuvo como objetivo principal determinar los niveles de competencia científica que alcanzan los estudiantes desde la estrategia de resolución de problemas a través de los trabajos prácticos de laboratorio articulados al paradigma pedagógico ignaciano. La pedagogía ignaciana tiene por clave y razón de ser el enseñar a pensar y enseñar a aprender, tanto en el ámbito científico-cultural como en el ámbito humano-cristiano (Jesuitas Educsi s.f.). Esta pedagogía contempla cinco pasos: contexto, experiencia, reflexión, acción y evaluación; así, sugiere una multitud de caminos en los que los educadores podrían acompañar a sus alumnos y facilitarles el aprendizaje y la madurez, enfrentándolos con la verdad y el sentido de la vida (Granados, 2005).

A través de esta articulación, el trabajo se enfocó en la relación de la química con la vida cotidiana con el fin de promover la investigación en la educación básica. Se utilizó el modelo de resolución de problemas a través de las prácticas de laboratorio, porque permite a los estudiantes adquirir habilidades de interpretación, argumentación, relación y participación (Coronel y Curotto, 2008). Por otro lado, se propusieron prácticas de laboratorio a partir de fenómenos cotidianos para entender su relación con la química y promover habilidades científicas desde las más sencillas hasta las más complejas. Con esto, los estudiantes adquirieron tanto las destrezas en el manejo instrumental, como las habilidades cercanas a la ciencia. El trabajo de laboratorio fundamentado en la resolución de problemas posibilitó mayores niveles de interés los cuales se pudieron evidenciar en un contexto real. 
Los instrumentos trabajados propiciaron el uso de habilidades para resolver problemas y orientaron al estudiante al uso de otras técnicas para la solución. Mediante una pregunta general se desarrolló un ejercicio de escritura que diera razón de lo que el estudiante podía relacionar del tema trabajado, generando una oportunidad de proponer mecanismos de solución mediante un proceso investigativo.

\section{Metodología}

Este trabajo de investigación adoptó los principios de la metodología mixta como lo menciona Onwuegbuzie y Johnson (2004): “Los métodos mixtos de investigación son formalmente definidos como una clase de investigación donde el investigador mezcla o combina técnica, métodos, aproximaciones, conceptos y lenguaje cuantitativos y cualitativos en un estudio simple". (Onwuegbuzie \& Johnson, 2004, p.17).

\section{Población y muestra}

Se trabajó en el Colegio Mayor de San Bartolomé, en el periodo 2014-2, con estudiantes de grado noveno para caracterizar las estrategias de aprendizaje en el área de ciencias naturales trabajadas hasta el momento y la actitud frente a las prácticas de laboratorio. En el periodo 2015-1 se continuó la investigación con estos estudiantes y se aplicó la estrategia pedagógica fundamentada en las situaciones problema a 38 de ellos, quienes se ubicaron en el curso 1004.

\section{Fases}

Este proyecto se realizó en tres fases, inicialmente se identificó la estrategia de enseñanza, posteriormente se crearon e implementaron las situaciones problema y finalmente se conoció el alcance de la estrategia. (Figura 1).

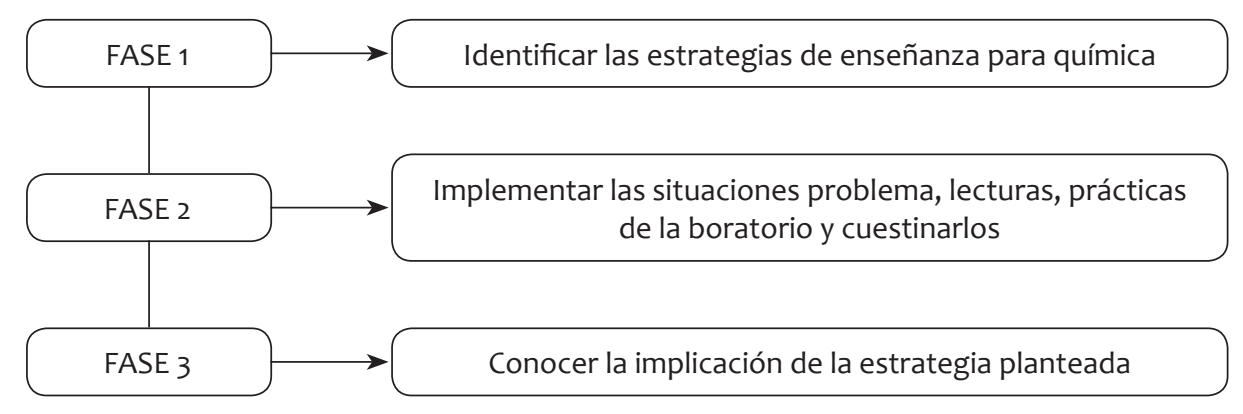

Figura 1 Fases del proyecto resolución de problemas a través de trabajos prácticos de laboratorio

Fuente: elaboración propia

\section{Instrumentos:}

- Test de caracterización: se indagó por las estrategias que los estudiantes habían tenido en clase de ciencias y las que querían tener en la clase de química.

- Situaciones problema: se realizaron dos situaciones problema, el primero tenía que ver con suplementos alternativos de calcio desde la cáscara de huevo, y el segundo consistía en agua potable. Estas se desarrollaron en las siguientes etapas: lectura, práctica de laboratorio y cuestionario.
Las situaciones problema se analizaron según los criterios establecidos en el cuadro 1 (Anexo 1) y el cuestionario a través de respuesta válida desde el criterio químico.

\section{Resultados y discusión}

Fase 1. Los resultados obtenidos evidenciaron el interés por parte de los estudiantes por los proyectos con fines de investigación, con un $68.4 \%$ de favorabilidad; así mismo, los laboratorios con fines de investigación con un $60,5 \%$, las prácticas de laboratorio y la resolución de problemas tuvieron 
un $47.4 \%$ de interés, lo que reflejó el interés de los estudiantes por los trabajos prácticos, donde el aula de clase se convierte en un ambiente práctico formador de conocimiento. A su vez, se ponen a prueba técnicas de experimentación y se desarrolla el quehacer científico para resolver situaciones problema de manera grupal o individual.

Fase 2. Situación problema 1: Suplementos alternativos de calcio desde la cáscara de huevo (Anexo 2).

La situación se planteó desde la temática de funciones químicas, los estudiantes ya conocían la formación de óxidos, bases, ácidos y las sales, y las diferentes reacciones que tienen. Se pretendió que ellos conocieran una aplicación de un desecho diario como la cáscara de huevo que contiene carbonato de calcio, con el fin de que ellos lograran crear suplementos de calcio usando este producto, a partir de la aplicación de sus conocimientos teóricos y prácticos y así lograr resolver la pregunta problema: ¿Cómo puede aportar la cáscara de huevo a la deficiencia de calcio en el mundo?

Se analizaron a continuación los ítems más importantes de las tres etapas:

La pregunta "¿cómo podrías reciclar la cáscara de huevo?" permitió que el estudiante pueda hacer una planificación desde el inicio, lo que lo condujo a solucionar la situación. Los resultados revelan que en el nivel 1 y 2 no hay ningún estudiante, en el nivel 3 hay 24 estudiantes que llegan a conclusiones basadas en investigaciones simples, son capaces de seleccionar hechos y conocimientos para explicar fenómenos y aplicar modelos sencillos y de utilizar y aplicar conceptos químicos básicos, mencionan "triturar la cáscara de huevo y agregarla a la comida para consumirla"; son capaces de razonar y de realizar interpretaciones de resultados. En el nivel 4 hay 14 de estudiantes que fueron capaces de seleccionar e integrar conocimientos desde diferentes disciplinas, ya que respondieron a la pregunta con la explicación "Agregarle la cáscara a alimentos para que tenga una mejor absorción en el cuerpo". También hicieron alusión a que la cáscara se puede utilizar como abono para las plantas por el contenido de calcio que posee, y para actividades artísticas. Se evidenció el uso y la aplicación de conceptos científicos. En los niveles 5 y 6 no se encontraron estudiantes, ninguno respondió con una explicación científica consistente.

\section{Gráfica 2 Porcentaje de respuesta, Nivel de competencias Científicas: Suplementos de calcio}

En la parte experimental los estudiantes debían crear los suplementos de calcio. La pregunta orientadora fue: ¿cómo se pueden crear suplementos de calcio a partir de la cáscara de huevo? 29 del total de estudiantes tienen un nivel de competencia 2, ya que ellos crearon los suplementos de calcio a partir del limón, vinagre, ácido nítrico o ácido clorhídrico, se evidenció que los estudiantes tenían el conocimiento adecuado sobre reacción química, llegaron a interpretar el fin de la práctica. Sin embargo, se les dificultó generar el nombre del compuesto porque no relacionaron las propiedades de los ácidos cítrico y acético con la formación de las sales y con las normas de nomenclatura; no identificaron la formación de una sal orgánica. La explicación del fenómeno es más macroscópica que desde aspectos microscópicos y químicos de la reacción.

Cuatro de los estudiantes estuvieron en un nivel 3; ellos lograron hacer la relación, emplearon los conceptos de química orgánica e inorgánica para la formación de la sal y nombraron correctamente los compuestos que elaboraron para utilizarlos como suplementos. La descripción de la reacción la hicieron desde un enfoque macroscópico de la materia y de lo observado. Tampoco se evidenciaron estudiantes en los niveles 4,5 y 6 de 
competencias, ya que no lograron usar un lenguaje científico y las explicaciones carecen de un nivel complejo de argumentación.

En el cuestionario las preguntas 1 a la 9 hacían referencia a la articulación de la teoría con las actividades prácticas que se realizaron en el laboratorio para la formación del carbonato de calcio. Los resultados muestran que en las preguntas 4,7 y 8 , referidas a la formación de las funciones inorgánicas ácidos y sales, 22, 29 y 29 estudiantes respectivamente no dieron respuestas correctas, lo que permite inferir dificultad para entender la temática, que no es clara, a pesar de haber sido recientemente trabajada. En las preguntas 1, 2, 5, 6 y 9 el porcentaje de asertividad fue mayor en un rango de 28 a 38 estudiantes, lo que evidenció que la formación de óxidos se les facilitó a los estudiantes; así mismo, los métodos de separación y las fuentes de calcio.

En la pregunta problema “¿cómo puede aportar la cáscara de huevo a la deficiencia de calcio en el mundo?", 32 estudiantes se ubicaron en el nivel 2, ya que manifestaron que la cáscara de huevo sí incrementarían los niveles de calcio, pero no de la misma manera que lo haría un suplemento dietario, debido a su proceso de elaboración. Se evidenció la influencia social y cultural que los medios de comunicación ejercen en la toma de decisiones y la construcción del conocimiento por parte de los estudiantes. En el nivel 3, seis estudiantes manifestaron que la cáscara de huevo hay que utilizarla como fuente de calcio y utilizar diferentes bebidas como el jugo de limón o de naranja; sin embargo, a las respuestas les faltaba argumentación desde los conceptos químicos involucrados. De lo anterior se infiere que el avance en la competencia interpretativa y reflexiva no ha sido de carácter significativo, no ha tenido una mayor relevancia en el desarrollo del proceso para resolver la situación problema.

Situación problema 2: Agua potable (Anexo 2).

Este instrumento se fundamentó en la lectura inicial retomada de las pruebas PISA (Programa Internacional para la Evaluación de Estudiantes) del año 2006; este enfoque se seleccionó ya que articula la temática de procesos de separación de mezclas estudiado por los estudiantes recientemente.
Se hizo una modificación a la prueba original, agregándose un texto de contextualización. Esta situación buscó que los estudiantes analizaran la calidad del agua del colegio y conocieran sus condiciones para que luego buscaran la solución a la descontaminación de esta. Las preguntas problema fueron: “¿El agua que llega al colegio reúne las condiciones de potabilidad que posee cuando sale de la planta de purificación?”, y “¿cómo se puede descontaminar el agua qué tiene microorganismos?". A continuación, se presentó el análisis de los ítems más importantes de las tres etapas:

Los estudiantes debían dar una explicación de por qué hay menos bacterias y partículas contaminantes en las aguas subterráneas que en las aguas de la superficie, como las de lagos y ríos. Se identificó que tres de los estudiantes están en un nivel 1 de competencias científicas porque no hay respuesta al interrogante, ningún estudiante estuvo en los niveles 2 y 3 de competencia. En el nivel 4 hay 23 estudiantes, ellos fueron capaces de seleccionar e integrar explicaciones y relacionarlas directamente con situaciones de la vida real; hubo respuestas como "el agua subterránea está dentro de la tierra y por lo tanto la contaminación del aire no puede ensuciarla, porque el agua subterránea no está al aire libre, está localizada en el fondo"; "los lagos y los ríos pueden ser contaminados por el aire", y "el agua subterránea es un agua sin muchos nutrientes para las bacterias, por eso no sobrevivirán en ella". En los niveles 5 y 6 hay 10 y 2 estudiantes respectivamente cuyo nivel de competencias científicas es alto, los estudiantes identificaron, explicaron y aplicaron el conocimiento científico a la situación problema, relacionaron distintas fuentes de información y de explicaciones para justificar desde su conocimiento teórico las respuestas.

Generalmente mencionaron que cuando el agua pasa a través de la arena se limpia, es decir, que ha tenido un proceso de filtración natural. Cuando el agua desciende a través del suelo, será filtrada por las rocas y la arena. Estas respuestas permiten establecer relación con lo planteado por García y García (2000),

"Problematizar algún aspecto de la cotidianidad o del entorno ambiental del estudiante y con base en ello, diseñar una estrategia completamente 
innovadora para la enseñanza de contenidos químicos en un aula mediada por un contexto determinado, hace que los estudiantes apliquen el conocimiento a la realidad." (García y García; 2000, p.34),

Sobre la pregunta problema del laboratorio “¿cómo se puede descontaminar el agua que tiene microorganismos e impurezas?" se resolvió que, desde la parte práctica y teórica, los estudiantes crearan sus propias estrategias de descontaminación. Se evidenció que en el nivel 1 se encuentran cinco de los estudiantes, ellos respondieron cosas de manera sencilla como hervir el agua, aplicaron el conocimiento cotidiano (situaciones familiares), sin dar una construcción compleja de la estrategia práctica a la purificación del agua. Ocho de los estudiantes utilizaron el método de añadirle cloro al agua y usar la ebullición para eliminar las bacterias, llegaron a construir un método de solución sencillo, pero únicamente abordaron lo relacionado con los microorganismos patógenos. En el nivel tres 15 estudiantes plantearon primero realizar una filtración con el papel correspondiente, luego agregar cloro y después someter el agua a ebullición. Se evidenció la falta de relación entre solubilidad de los gases y la temperatura; sin embargo, se identificó que los estudiantes relacionaron conocimientos teórico-prácticos para aplicar modelos sencillos y estrategias de investigación, lograron utilizar y aplicar conceptos científicos.

Diez de los estudiantes tuvieron un mayor nivel práctico para la solución de la problemática. Ellos hicieron lo mismo que los compañeros del nivel 3; sin embargo, volvieron a observar cómo estaba el agua en las nuevas condiciones, para saber si su método había funcionado. Fueron capaces de seleccionar e integrar explicaciones de diferentes disciplinas de ciencia como la biología y la química, y relacionarlas directamente con situaciones de la vida real. Para Gaulin (2001) estas situaciones problema demandan reflexión, búsqueda, investigación, y para responder hay que pensar en las soluciones y definir una estrategia de resolución que no conduce, precisamente, a una respuesta rápida e inmediata.

En el cuestionario la pregunta 5 se articulan los reactivos que se utilizan en el laboratorio con su fórmula molecular. Treinta y tres estudiantes respondieron de una manera acertada la formación de las sales y el ácido utilizado, lo que evidenció que ha ocurrido mejora; sin embargo, a cinco estudiantes se les dificultó.

Para los siguientes interrogantes "¿Cuál es la calidad del agua del colegio?”, “qqué medidas se podrían tomar para que mejore?”, dieciocho de los estudiantes se ubicaron en el nivel de competencia, 3 y 15 estudiantes en el nivel 4. Estos niveles son en su medida buenos ya que hay una explicación coherente y su dominio conceptual sobre los métodos de separación para purificar el agua son buenos. Sin embargo, el nivel de argumentación es bajo, se limitan a escribir de una manera muy simple, sin utilizar el lenguaje científico.

Se evidenció que 5 estudiantes, al analizar esta situación, demostraron una verdadera comprensión del problema, y pudieron llegar a la solución de la misma, por lo tanto, se ubican en el nivel 5. En este nivel los estudiantes evidenciaron relaciones conceptuales desde la parte química y biológica para la solución del interrogante; se infiere que los estudiantes comienzan a ser más reflexivos y han empezado a dar respuestas más elaboradas desde un marco teórico fundamentado. Lo anterior evidencia que considerar el aprendizaje como una construcción social que incluye conjeturas, pruebas y refutaciones con base en un proceso creativo y generativo, como lo plantea Coronel y Curotto (2008), y con la incorporación de las prácticas de laboratorio, hace que se logre un aprendizaje más efectivo de los diferentes conceptos y se relacione con la solución a situaciones problema cotidianas.

\section{Conclusiones}

Se logró un aprendizaje significativo de funciones químicas y métodos de separación desde la estrategia de resolución de problemas, a través de los trabajos prácticos de laboratorio. Así mismo, se llegó a las siguientes conclusiones:

- Se evidenció la evolución de los estudiantes en el nivel adquirido de competencia 
científica, competencias investigativas, resolución y análisis de problemas, uso de la experimentación, relación de los fundamentos teóricos y los trabajos prácticos articulados a la resolución de las diferentes situaciones problema.

- Se identificaron inicialmente las actitudes favorables por parte de los estudiantes frente a las prácticas de laboratorio y la resolución de problemas.

- Los estudiantes diseñaron la propuesta de laboratorio, que les permitió lograr aprendizajes significativos como también sentirse capaces de generar o construir su propio conocimiento, actitudes que se evidenciaron durante el desarrollo de la estrategia.

\section{Referencias}

Coronel M., y Curotto M. (2008). La resolución de problemas como estrategia de enseñanza y aprendizaje. Revista Electrónica de Enseñanza de las Ciencias, 7 (2), 463-479.

Galindo Rodríguez, I. (2011). La resolución de problemas: Una perspectiva desde el aprendizaje por investigación. PPDQ Boletín, (47), 37-47.

García, J., y García, F. (2000). Aprender investigando: Una propuesta metodológica basada en la investigación. Sevilla: Díada editorial.

Gaulin, C. (2001). Tendencias actuales de la resolución de problemas. Sigma, (19), 51-63. Recuperado de https://sferrerobravo.files.wordpress. com/2007/10/7_tendencias_actuales.pdf

Granados L. (2005). Paradigma pedagógico ignaciano. Reflexiones educativas y pedagógicas con inspiración ignaciana. Recuperado de http://www.acosicam.com/sites/default/files/acosicam_paradigma_ pedagogico_ignacio.pdf

Jesuitas Educsi (s.f.). La Pedagogía Ignaciana. Recuperado de http://www.educacionjesuitas.es/ identidad/la-pedagogia-ignaciana

Onwuegbuzie, A., y Johnson, B. (2004). Mixed Methods Research: A Research Paradigm whose Time has come. Educational Researcher, 33 (7), 14-26.

Pisa. (2009). PISA: COMPETENCIA CIENTÍFICA. Edita: ISEI.IVEI Instituto Vasco de Evaluación e Investigación Educativa. p. 15. 


\section{Anexo 1}

\section{Cuadro 1. Niveles de competencia científica de PISA, 2009, resumen}

\begin{tabular}{|c|l|}
\hline Nivel & \multicolumn{1}{|c|}{ Tareas } \\
\hline 6 & $\begin{array}{l}\text { Los estudiantes identifican, explican y aplican el conocimiento científico en diversas situaciones complejas de la } \\
\text { vida real. Relacionan distintas fuentes de información y explicaciones y utilizan pruebas de estas fuentes para } \\
\text { justificar decisiones. }\end{array}$ \\
\hline 5 & $\begin{array}{l}\text { Pueden identificar los componentes científicos de muchas situaciones complejas de la vida real, aplicando a } \\
\text { ellas conceptos científicos. Son capaces de utilizar capacidades de investigación bien desarrolladas y relacionar } \\
\text { el conocimiento adecuadamente. Son capaces de elaborar explicaciones basadas en pruebas y argumentos } \\
\text { basados en su análisis crítico. }\end{array}$ \\
\hline 4 & $\begin{array}{l}\text { Son capaces de seleccionar e integrar explicaciones de diferentes disciplinas de ciencia y tecnología, y } \\
\text { relacionarlas directamente con situaciones de la vida real. }\end{array}$ \\
\hline 3 & $\begin{array}{l}\text { Pueden identificar cuestiones científicas descritas claramente. Son capaces de seleccionar hechos y } \\
\text { conocimientos para explicar fenómenos y aplicar modelos sencillos o estrategias de investigación, y de utilizar y } \\
\text { aplicar conceptos científicos. }\end{array}$ \\
\hline 2 & $\begin{array}{l}\text { Pueden llegar a conclusiones basadas en investigaciones simples. Son capaces de razonar y de realizar } \\
\text { interpretaciones literales de resultados de una investigación científica. }\end{array}$ \\
\hline 1 & $\begin{array}{l}\text { Solo pueden aplicar el conocimiento científico a unas pocas situaciones familiares. Son capaces de presentar } \\
\text { explicaciones científicas obvias que se derivan explícitamente de las pruebas dadas. }\end{array}$ \\
\hline
\end{tabular}

Tomado de Pisa. (2009). PISA: COMPETENCIA CIENTÍfICA. Edita: ISEI.IVEI Instituto Vasco de Evaluación e Investigación Educativa. 


\section{Anexo 2}

¿CÓMO PUEDE APORTAR LA CÁSCARA DE HUEVO A LA DEFIIENCIA DE CALCIO EN EL MUNDO? Para ello se realizaran pruebas en el laboratorio para conocer más sobre las propiedades que tiene.

\section{objetivos}

- Identificar propiedades del carbonato de calcio (cáscara de huevo)

- Realizar suplemento de calcio desde la cáscara de huevo

1. Experimentación

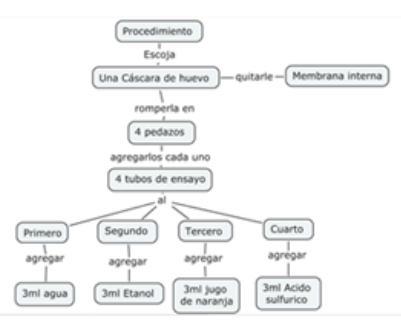

SUPLEMENTOS DE CALCIO DESDE LA CASCARA

Desde lo anterior se puede observar en qué tipo de sustancias la cáscara de huevo es soluble, por lo cual se pueden hacer los suplementos alternativos con sustancias de carácter similar.

¿CÓMO A PARTIR DE LA CÁsCARA DE HUEVO SE PUEDE CREAR SUPLEMENTOS DE CALCIO? A partir de la cascara de huevo elabora tres suplementos de calcio.

PREPARACIÓN DE SUPLEMENTOS

\begin{tabular}{|c|l|l|l|}
\hline SUPLEMENTO & & & \\
\hline $\begin{array}{c}\text { REACTIVO } \\
\text { UTIUZADO }\end{array}$ & & & \\
\hline OBSERVACIONES & & & \\
\hline REACCÓN & & & \\
\hline
\end{tabular}

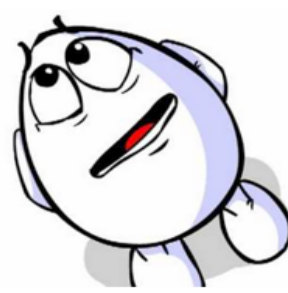

FUENTE DESPERDICIADA DE CALCIO

En un importante congreso celebrado en Roma, científicos de todo el mundo subrayaron la necesidad de consumir alimentos ricos en calcio y vitamina $D$, como mejor sistema para asegurar la ingestión adecuada de estos elementos y ayudar a evitar problemas como formación ósea o crecimiento deficiente. En el I Congreso Mundial sobre el Calcio y la Vitamina D en la Vida Humana, organizado por la FAO, la Organización Mundial de la Salud (OMS) y otras instituciones dedicadas a la nutrición, se discutieron las formas de mejorar el consumo de estos elementos nutritivos de todos los grupos de la población, y promover un crecimiento adecuado durante la lactancia, la niñez y la adolescencia.

Un consumo insuficiente de calcio se asocia en todo el mundo a diversos problemas médicos comunes de carácter crónico, tales como la osteoporosis, enfermedades cardiovasculares: hipertensión, infartos y males isquémicos del corazón, diabetes y problemas de hipertensión durante la gestación, obesidad y cáncer del colon. Así mismo el calcio también participa en numerosos procesos metabólicos, como la contracción muscular, los procesos mediados por enzimas, la liberación de hormonas y neurotransmisores y la coagulación sanguínea.

En todo el ciclo vital, el calcio y la vitamina $D$ son esenciales para un buen crecimiento y para la salud. Tienen especial importancia durante los periodos de crecimiento rápido de la niñez y la adolescencia, asi como durante el embarazo y la lactancia.

En el mundo 870 millones de personas tienen malnutrición calórica, a los que hay que añadir unos dos mil millones con déficit de calcio. Según la FAO, el $26 \%$ de los y las menores de cinco años presentan retraso del crecimiento debido a la falta de este. Además los recursos económicos de algunas poblaciones suelen ser muy limitados, incluso inexistentes; además cuando hay escasez frecuente los precios de la leche y cereales aumentan, hace que estas familias se encuentren en pobreza y hambre crónica, que afecta a todos los miembros de la familia pero especialmente a las mujeres embarazadas y a la infancia. Así mismo buena parte de la población no los consume por intolerancia.

Cobra mayor importancia porque el $99 \%$ de la masa ósea es calcio y $45 \%$ se forma en la ADOLESCENCIA, en esta etapa se debe acopiar mayor cantidad de calcio para evitar fracturas y osteoporosis en la adultez. Se recomiendan unos $1200 \mathrm{mg} /$ día. El calcio más importante es el ingerido y acumulado durante edades tempranas, por ser ese el periodo en el cual se produce la mayor formación de hueso. Cuando el organismo para funcionar equilibradamente no lo obtiene de los alimentos, lo quita de los huesos, los que se descalcifican gradualmente, los debilita y vuelve frágiles y quebradizos, hasta llegar a una enfermedad que se conoce como osteoporosis.

En el mercado se venden suplementos para tratar las deficiencias de calcio, los dos tipos principales de este mineral son el carbonato de calcio y el citrato de calcio. Ambos tipos de suplementos le proporcionarán al cuerpo suficiente calcio. 
